\title{
Die Korrespondenz Einstein-Schlick: Zum Verhältnis der Physik zur Philosophie
}

\author{
Klaus Hentschel \\ Cankebeer str. 97, 2981 Westdorf, F.R. Germany
}

Received 1 September 1985

\section{Zusammenfassung}

Es wird die wechselseitige Beeinflussung Einsteins und Schlicks anhand ihrer ab 1915 erhaltenen Korrespondenz in vier Schwerpunkten untersucht. Schlicks Selbstverständnis als Philosoph wie auch einzelne Themata seines Denkens (wie etwa das der Einfachheit) bildeten sich mit seiner Auseinandersetzung um die Relativitätstheorie Einsteins heraus, deren systematische Explikation durch Schlick auf Einsteins Beifall stieß. Als die Ursache für das Auseinanderdriften beider Denker nach 1925 werden fundamentale Differenzen im Wirklichkeitsverständnis und in der Interpretation des Kausalitätsprinzips aufgewiesen, die beide auch zu komplementären Formen der Wissenschaftsgeschichtsbetrachtung führten.

\footnotetext{
Summary

Some topics in the Einstein-Schlick correspondence are discussed and embedded in the historical and scientific context of the years 1915-1936.

Different interpretations of the theory of relativity and their relation to Schlick's papers on relativity. It is shown that Einstein welcomed Schlick's interest in relativity because of Schlick's talent to popularize without simplifying, and that Schlick's selfassessment as a 'Sinndeuter' (clarifier of sense) of results of science developed in the course of his studies in relativity.

Simplificity as a central theme in Einstein's thought. It is demonstrated, that their exchange of thoughts intensified after Schlick adopted the motiv of simplicity; nevertheless, Schlick realized quite quickly that criteria of 'simplicity' have pragmatic and aesthetic components, while Einstein continued to believe in the logical unequivocality of simplicity.

Different concepts of reality. An interesting phase in Einstein's philosophical development, a sort of 'epistemological neutralism' or 'conventionalism', is discussed in connection with Einstein's confrontation of different possible uses and meanings of reality.

Newton and causality. Einstein's justification of Newton's introduction of the concept of the principle of causality, and their models for the progress of science, are presented.
}

Inhalt

1. Einleitung ................................................ 476

2. Interpretationen der Relativitätstheorie $\ldots \ldots \ldots \ldots \ldots \ldots \ldots \ldots \ldots \ldots, 476$

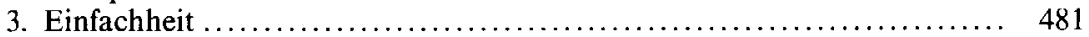

4. 'Wirklich' in verschiedenem Sinne ................................. 483

5. Newton und die Kausalität .................................... 485 


\section{Einleitung}

Trotz eines seit dem Einstein-Jubiläum 1979 unverändert großen Interesses einer breiten Öffentlichkeit für die Person ${ }^{1}$ und das Werk Albert Einsteins ${ }^{2}$ und einer unlängst wiedererwachten Aufmerksamkeit gegenüber dem Schlickschen Philosophieund Naturwissenschaftsverständnis, ${ }^{3}$ wurde bis jetzt nichts detailliertes bekannt über die Korrespondenz beider, die zu großen Teilen erhalten geblieben ist und in Kopien sowohl im Einstein-Nachlaß als auch im Schlick-Nachlaß vorhanden ist. ${ }^{4}$

Da beide Korrespondenten gleichermaßen als Physiker wie als Philosoph angesprochen werden könnten, läßt sich schon von daher einiges Interessantes über das Verhältnis physikalischer Grundlagenforschung und philosophischer Reflexion erwarten. Die nähere Beschäftigung mit der Korrespondenz erweist darüber hinaus, daß ferner eine Unzahl von naturphilosophischen, wissenschaftshistorischen bzw. -philosophischen Fragen in der Korrespondenz berührt sind, die jede für sich genommen bereits eine ausführlichere Darlegung und Präsenz in der bis heute über diese Fragen andauernden Diskussion verdienten. ${ }^{5} \mathrm{Im}$ folgenden wurden deshalb einige geeignete Themenschwerpunkte aus ihrem umfangreichen Kontext herausgelöst und durch relevante Hintergrundinformationen ergänzt.

\section{Interpretationen der Relativitätstheorie}

Albert Einstein begann den ersten erhaltenen Brief seiner Korrespondenz mit Moritz Schlick (vermutlich der erste schriftliche Kontakt zwischen beiden überhaupt) mit den folgenden Worten über Schlicks Aufsatz 'Die philosophische Bedeutung des Relativitätsprinzips' (1915): 'Ich habe gestern Ihre Abhandlung erhalten und bereits vollkommen durchstudiert. Sie gehört zu dem Besten, was bisher über Relativität geschrieben worden ist. Von philosophischer Seite scheint überhaupt nichts annähernd klares über den Gegenstand geschrieben worden zu sein.'(14 Dezember 1915). Dies war

${ }^{1}$ Vgl. u.a. die Einstein-Biographien von Bernstein, Clark, Dukas-Hofmann, Frank, Herneck, Pais, Schlicker, Seelig u. Wickert sowie D. C. Cassidy, 'Biographies of Einstein', in Einstein-Symposium Berlin (Berlin, 1979), S. 490-500.

${ }^{2}$ Vgl. u.a.: Albert Einsteins Einfluß auf Physik und Politik, hrsg. P. C. Aichelburg u. R. U. Sexl (Braunschweig u. Wiesbaden, 1979); Albert Einstein - historical and cultural perspectives, hrsg. Y. Elkana u. G. Holton (Princeton, 1982); G. Holton, Thematische Analyse der Wissenschaft (Frankfurt, 1981); G. Holton, Themata (Braunschweig, 1984); Einstein-Symposium Berlin (Anm. 1), hrsg. H. Nelkowski et al. Alhert Einstein als Philosoph und Naturforscher, hrsg. A. P. Schilpp (engl. Original 1949, dt. Übers.: Braunschweig, 1979).

${ }^{3} \mathrm{Vgl}$. die folgenden Reprints von Originalarbeiten Schlicks: M. Schlick, Philosophical Papers, hrsg. H. Mulder u. B. van de Velde-Schlick (Dordrecht u. Boston, Vol. I (1978), Vol. II (1979); M. Schlick, Allgemeine Erkenntnislehre (1. Aufl. 1918, 2. erweiterte Aufl. 1925, Reprint Frankfurt, 1979); ders., Fragen der Ethik (1. Aufl. 1930, Reprint Frankfurt, 1984); ders., Die Probleme der Philosophie in ihrem Zusammenhang (Vorlesungen im Wintersemester 1933-34) (Frankfurt, 1986). Vgl. ferner: H. Rutte, 'Moritz Schlick, der Positivismus und der Neo-positivismus', Zeitschrift für philosophische Forschung, 30 (1976), 246-68; H. D. Finke, Methode und Aufgabe der theoretischen Philosophie und die Genese des Sinnkriteriums hei Moritz Schlick (Hamburg, 1980); Zurück zu Schlick, hrsg. B. McGuinness (Wien, 1985).

${ }^{4}$ Reuven Yaron, Einstein Archival Materials at the Hebrew University Library, Jerusalem, document call Arc.4 1576/reel 21; John Stachel, The Collected Papers of Alhert Einstein (Boston University); Henk Mulder u. A. J. Knox, Vienna Circle Foundation (Amsterdam).

${ }^{5}$ Vgl. K. Hentschel, 'Zum Verhaltnis Philosophie-Physik anhand der Korrespondenz Schlick-Einstein und ergänzender Dokumente' (Magisterarbeit Universität Hamburg, 1984); D. Howard, 'Realism and Conventionalism in Einstein's Philosophy of Science: The Einstein-Schlick-Correspondence', Philosophia Naturalis, 21 (1984), 616-29.

${ }^{6}$ Schlick, 'Die philosophische Bedeutung des Relativitätsprinzips', Zeitschrifi für Philosophie und philosophische Kritik, 159 (1915), 129-75. 
kein geringes Kompliment für den damals noch völlig unbekannten Rostocker Privatdozent Schlick. Um diese Lobesworte Einsteins zu erläutern, sei mir ein kurzer Rückblick auf die frühe Rezeption der Relativitätstheorie ${ }^{7}$ gestattet.

Die Relativitätstheorie in ihrer speziellen Form geht zurück auf Einsteins berühmte Arbeit 'Zur Elektrodynamik bewegter Körper' $(1905)^{8}$ und war beschränkt auf die geforderte Äquivalenz der Beschreibung elektromagnetischer Prozesse in geradlinig gleichförmig zueinander bewegten Bezugssystemen. Fünf Jahre lang war sie nur in Expertenkreisen (spez. von der Sommerfeld-Schule in München) ${ }^{9}$ zur Kenntnis genommen worden, dann erschienen (ab 1910) erste populäre Aufsätze, zunächst vorwiegend von Physikern (Planck, Langevin, Berg...), ${ }^{10}$ durch die die zentralen physikalischen Argumente und Postulate Einsteins allgemein bekannt wurden. Dass darin einige ihrer ungewöhnlichen, scheinbar paradoxen Konsequenzen durch drastische Beispiele illustriert wurden, (insb, das berühmte Zwillingsparadoxon ${ }^{11}$ ), hat später entgegen der Absicht dieser Autoren, viel zur politisch-weltanschaulichen Aufheizung der Debatte beigetragen ${ }^{12}$. Bald darauf begann eine sich bis 1925 zur Veröffentlichungsfiut ausweitende Serie von Publikationen aus der Feder von Philosophen verschiedenster Schulzugehörigkeit. ${ }^{13}$ Diese Publikationen wurden verfasst in der Absicht

entweder, ihr jeweiliges 'Credo' zu immunisieren gegenüber den neuen physikalischen Setzungen und Hypothesen

oder, um Verträglichkeit bzw. gar einen historischen Entstehungszusammenhang zwischen ihren philosophischen Prämissen und der Relativitätstheorie nachzuweisen.

Im Gegensatz zu diesen Schriften war das für Einstein hervorstechende und lobenswerte an Schlicks Darstellung der 'philosophischen Bedeutung der Relativitätstheorie', daß in seinem Aufsatz die zahlreichen Mißverständnisse, Verzerrungen und eigentümlichen Metamorphosen der Gedankengänge der Relativitätstheorie vermieden waren und Einsteins Selbstverständnis zutreffend erfasst worden war. In der folgenden Auflistung der Punktc. dic Finstein an Schlicks Aufsatz besonders

\footnotetext{
${ }^{7}$ Vgl.z.B. A.I.Miller, Albert Einstein's special theory of relativity-emergence (1905) and early interpretation (1905-1911) (Reading, Mass., 1981), sowie Hentschel (Anm. 5, 1984), Kap.2 u.3.

${ }^{8}$ A. Einstein, 'Zur Elektrodynamik bewegter Körper', Annalen der Physik, (4), 17 (1905), 891-921 und zahlreiche Reprints, z.B. in H. A. Lorentz, A. Einstein U. H. Minkowski, Das Relativitätsprinzip (Stuttgart, 7. Aufl. 1974).

${ }^{9}$ Vgl. A. Einstein u. A. Sommerfeld, Briefwechsel ..., hrsg. A. Hermann (Basel u. Stuttgart, 1968).

${ }^{10}$ M. Planck, Acht Vorlesungen über theoretische Physik (Leipzig, 1910); P. Langevin, 'L'évolution de l'espace et du temps', Scientia, 10(1911), 31-54 sowie ders., 'Le temps, l'espace et la causalité dans la physique moderne', Bulletin de la Société Francaise de Philosophie, 12 (1912), 1-45 (inkl. Diskussion mit Rey, Perrin, Milhaud, Le Roy, Borel, Brunschvicg u. Darlu); O. Berg, 'Das Relativitätsprinzip der Elektrodynamik', Abhandlungen der Friesschen Schule (Göttingen), (2), 3 (1910), 333-82.

${ }^{11} \mathrm{Vgl}$. L. Marder, Reisen durch die Raum-Zeit; das Zwillingsparadoxon-Geschichte einer Kontroverse (Braunschweig, 1979).

${ }^{12}$ Vgl.u.a.S. Grundmann in Relativitätstheorie und Weitanschauung ...(Berlin (Ost), 1967); A. Hermann, 'Der Kampf um die Relativitätstheorie'. Bild der Wissenschaft (Stuttgart, 1977), S. 10916.

${ }^{13}$ Vgl. K. Hentschel (Anm. 5, 1984), Kan. 3 u. Bibliographie Teil III, 3
} 
bemerkenswert fand, ist zugleich etwas von dem Reflex des Stellenwertes eingefangen, den Einstein selbst 1915 seiner Theorie zugewiesen hat (14 Dezember 1915):

Das Verhältnis der Relativitätstheorie zur Lorentzschen Theorie ist ausgezeichnet dargelegt, wahrhaft meisterhaft das Verhältnis zur Lehre Kants und seiner Nachfolger. Das Vertrauen auf die 'apodiktische Gewißheit' der 'synthetischen Urteile a priori' wird schwer erschüttert durch die Erkenntnis der Ungültigkeit nur eines einzigen dieser Urteile. Sehr richtig sind auch Ihre Ausführungen darüber, dass der Positivismus die Relativitätstheorie nahe legt, ohne sie indessen $\mathrm{zu}$ fordern.

Auf die in diesem Zitat zum Ausdruck kommende Haltung Einsteins gegenüber dem Kantianismus einzugehen, ist hier nicht der Ort. ${ }^{14}$ Wesentlich scheint mir aber die für die Frage der erkenntnistheoretischen Quellen Einsteins entscheidende Präzisierung des Verhältnisses von Einstein zum Positivismus, die sich in folgenden Worten manifestiert (ibid):

Auch darin haben Sie richtig gesehen, dass diese Denkrichtung von grossem Einfluß auf meine Bestrebungen gewesen ist, und zwar E. Mach ${ }^{15}$ und noch viel mehr Hume, ${ }^{16}$ dessen Traktat über den Verstand ${ }^{17}$ ich kurz vor Auffindung der Relativitätstheorie mit Eifer und Bewunderung studierte. Es ist sehr gut möglich, dass ich ohne diese philosophischen Studien nicht auf die Lösung gekommen wäre.

Diese Zeilen sind der deutlichste mir bekannte Beleg für die geistige Nähe, in die Einstein (um 1915) sich selbst relativ zu Mach und Hume gerückt hat, und mehr noch für die überaus enge Bezichung, in die 'philosophische Studien' und 'physikalische Resultate' hier gerückt werden. Über alle noch anzusprechenden Änderungen der philosophischen Prämissen und Selbsteinschätzungen Einsteins hinweg, änderten sich seine grundsätzliche Offenheit gegenüber philosophischen Studien, seine Tendenz zum Rekurs auf philosophische Argumente als Stützung physikalischer Gedankengänge und insgesamt gesehen die Untrennbarkeit der Rollen 'Physiker' und 'Philosoph' Einstein nicht. ${ }^{18}$ Nichts desto trotz behielt Einstein gegenüber den Thesen aller traditoneller Philosophenschulen zur Relativitätstheorie den Vorbehalt, daß diese in das jeweilige Denksystem 'hineingezwängt' würde, ${ }^{19}$ während Schlicks Darstellung (geprägt von Sachverstand und einem zunächst 'absichtslosen' sich-auf-die-TheorieEinlassen) das Ergebnis eines gewandelten Verständnisses von Philosophie im Verhältnis zu den Einzelwissenschaften war, das zukunftsweisend werden sollte. Dieses, sich insb.

\footnotetext{
${ }^{14} \mathrm{Vgl}$. K. Hentschel, 'Einstein, Neokantianismus und Theorienholismus', eingereicht bei Kantstudien.

${ }^{15}$ Vgl. u. a. Fr. Herneck, 'Die Beziehungen zwischen Einstein und Mach dokumentarisch dargestellt', Wissenschaftliche Zeitschrift der Fr. Schiller Universität, Jena, 15 (1966), 1-14; G. Holton, 'Mach, Einstein und die wissenschaftliche Suche nach Realität', in Holton (Anm. 2), S. 203-54; J. Blackmore, K. Hentschel u. Fr. Wallner, 'Einstein und verschiedene Haltungen zur Philosophie und Physik Ernst Machs', eingereicht für eine Aufsatzsammlung von Fr. Stadler.

${ }^{16} \mathrm{Vgl}$. z. B. A. I. Miller, 'On Einstein's invention of special relativity, Philosophy of Science Association, 2 (1982); K. Hentschel (Anm. 5) Kap. 2.

${ }^{17}$ Es ist unsicher, ob Einstein hiermit Humes 'Traktat über die menschliche Natur' oder seine 'Untersuchung über den meschlichen Verstand' meinte-es spricht aber einiges für die erste Möblichkeit, da nur im 'treatise' ausführlichere Stellunghahmen Humes zu 'den Vorstellungen des Raumes und der Zeit' enthalten sind (vg1. 1. Buch, 2. Teil).

${ }^{18}$ Siehe den bezeichnenden Titel des Sammelbandes von Schilpp (1949-79, Anm. 2).

${ }^{19} \mathrm{Vgl}$. Brief Finstcins an Schlick vom 17 Oktober 1919.
} 
durch Schlicks Auseinandersetzung mit Einsteins Relativitätstheorie und ihrer Bedeutung für die Philosophie herausbildende ${ }^{20}$ (und nach der WittgensteinRezeption festigende ${ }^{21}$ ) Selbstverständnis Schlicks kann wie folgt grob umrissen werden: ${ }^{22}$

die Aufgabe der Naturwissenschaft wird bestimmt als die der Gewinnung von Erkenntnis über alle Naturvorgänge, d.h. die Aufstellung der wahren Sätze über sie und die ständig wiederholte Prüfung dieser Sätze einschließlich der Voraussetzungen, mittels derer diese Sätze formuliert werden können;

daneben gibt es nicht noch eine genuin philosophische Rechtfertigung oder Begründung der wissenschaftlichen Grundlagen, wohl aber eine als Tätigkeit verstandene Sinndeutung naturwissenschaftlicher Sätze. ${ }^{23}$

Freilich war Moritz Schlick auch geradezu prädestiniert zu dieser Rolle des gleichermaßen physikalischen Interpreten wie philosophischen Sinndeuters. 1882, also nur 3 Jahre nach Einstein, geboren, hatte er Physik in Heidelberg, Lausanne und Berlin studiert und 1904, also als 22-jähriger, bei Max Planck in Berlin mit einer Arbeit über die Reflexion des Lichtes in dünen, inhomogenen Schichten promoviert, sich danach aber philosophischen Fragen zugewandt und 1910 in Rostock über 'Wahrheitstheorien' habilitiert. ${ }^{24}$ Über seine Motive, die ihn zu den frühen populären Aufsätzen zur Relativitätstheorie führten, erfahren wir manches aus dem zweiten erhaltenen Brief der Korrespondenz von Schlick an Einstein von 4 Februar 1917. Anläßlich der Übersendung des Manuskriptes für einen von der Zeitschrift Die Naturwissenschaften erbetenen Aufsatz über 'Raum und Zeit in der gegenwärtigen Physik', das Schlick Einstein mit der Bitte um Korrektur beilegte, bemerkt Schlick unter Verweis auf das fremdbestimmte Thema (4 Februar 1917):

So ist der Aufsatz weniger eine Darstellung der allgemeinen Relativitätstheorie selbst als eine eingehende Erläuterung des Satzes, daß Raum und Zeit nun in der Physik alle Gegenständlichkeit eingebüsst haben. Mein Hauptziel war, die Darstellung so leicht verständlich zu machen wie irgend möglich. Es ist wirklich so sehr zu wünschen, dass die Gedanken des allgemeinen Rel(ativitäts)prinzips recht bald überall bekannt und verstanden würden, nicht bloß aus physikalischen, sondern auch besonders aus philosophischen Gründen.

\footnotetext{
${ }^{20}$ Neben Schlick (1915, Anm. 6); vgl. Schlick (1917, Anm. 6 bzw. 32) sowie M. Schlick, (Die Relativitätstheorie in der Philosophie', Verhandlungen der Gesellschaft deutscher Naturforscher und Ärzte, 87 (1922), und ders. 'Erkenntnistheorie und moderne Physik', Scientia, 45 (1929), 307-16 (verfasst bereits 1925).

${ }^{21}$ Zunchmend von Wittgenstein beeinflusste Aufsätze sind etwa: M. Schlick, 'Erleben, Erkennen, Metaphysik', Kantstudien, 31 (1926), 146-58; ders., 'Die Wende der Philosophie', Erkenntnis, 1 (1930/31), 4-11; vgl. Hentschel (Anm. 5), Kap. 14 u. Bibliographie III, 14.

${ }^{22} \mathrm{Vgl}$. etwa M. Schlick, 'Philosophie und Naturwissenschaft', Erkenntnis, 4 (1934), 79-99; ders., Grundzüge der Naturphilosophie (Wien 1948), S. 1 ff. (verfasst als Vorlesungsmanuskript, 1932-33).

${ }^{23}$ Der Einfluß Wittgensteins ist hier besonders deutlich. Dieser hatte nämlich in seinem Tractatus logicophilosophicus, erstmals in den Annalen der Naturphilosophie, 14(1921), 185-198 "die richtige Methode der Philosophie" mit den berühmten Worten umschrieben:... "Nichts zu sagen, als was sich sagen läßt, also Sätze der Naturwissenschaft - also etwas, was mit Philosophie nichts zu tun hat - und dann immer, wenn ein anderer etwas Metaphysisches sagen wollte, ihm nachzuweisen, daß er gewissen Zeichen in seinen Sätzen keine Bedeutung gegeben hat' (6.53) und hiermit Philosophie nicht mehr als System von Sätzen, dondern nur mehr als Tätigkeit verstanden.

${ }^{24}$ Zur Person Schlicks vgl.u.a.Schlicks Selbstdarstellung in W. Ziegenfuß u.G.Jung (Hrsg.), Philosophenlexikon, 2. Band (Berlin 1950), 462-464 sowie folgende Nachrufe: H. Feigl, 'Moritz Schlick', Erkenntnis, 7(1937/37), 393-419; v. Kraft, 'Moritz Schlick', Philosophia I, (Belgrad), (1936), 323-330 sowie D. Rynin, 'Schlick, (Friedrich Albert) Moritz', in Dictionary of Scientific Biography, Bd. 12(1975), S. 177-179.
} 
Beachtenswert ist, daß die Zielsetzung Schlicks, ebenso wie ein Hauptmotiv für das Interesse Einsteins am Popularisator Schlick darin bestanden, die Relativitätstheorie mit ihren zunächst theoretisch-physikalischen Aussagen Bestandteil der praktisch relevanten 'wissenschaftlichen Weltauffassung" ${ }^{25}$ werden zu lassen, und damit jenseits des dem logischen Empirismus immer wieder unterstellten rein-theoretischen Erkenntnisinteresses anzusiedeln sind. ${ }^{26}$

Ebenso wie Schlick muß auch ich hier auf eine ausführlichere Darlegung der allgemeinen Relativitätstheorie verzichten ${ }^{27}$, deren Kern in einer Ausweitung des Relativitätsprinzips auf alle Bewegungsvorgänge (also insb. auch beschleunigte) bestand. Die Naturgesetze sollen, so die Forderung der Allgemeinen Relativitätstheorie, gegenüber beliebigen Wechseln der Koordinatensysteme, die zur Naturbeschreibung herangezogen werden, ihre Form beibehalten (Kovarianz). Bei der Durchführung dieses Programms zeigte es sich, daß dies nur zu erreichen war durch die Einführung einer Krümmung des Raumes (Krümmungstensor), die abhängig gemacht wurde von der in seinem Umfeld vorhandenen Masse (Energie-Impuls-Tensor). Nachdem Einstein bereits 1911 (in der sog. Prager Theorie) ${ }^{28}$ als eine physikalisch nachweisbare Prognose die Lichtablenkung nahe der Sonnenoberfläche prophezeit hatte, ${ }^{29}$ wurde in den folgenden Jahren (bis 1916) mittels des Tensorkalküls die Theorie von Einstein zusammen mit Marcel Grossmann ausformuliert. ${ }^{30}$

In einer Seitenbemerkung von Einsteins erstem Brief an Schlick (14 Dezember 1915) kommentiert Einstein seine damals druckfrischen Resultate wie folgt: 'Das Neugefundene ist das Resultat, daß es eine mit allen bisherigen Erfahrungen vereinbare Theorie gibt, deren Gleichungen beliebigen Transformationen der Raum-ZeitVariablen gegenüber kovariant sind. Dadurch verlieren Zeit und Raum den letzten Rest von physikalischer Realität. Es bleibt nur übrig, dass die Welt als vierdimensionales (hyperbolisches) Kontinuum ... aufzufassen ist'. (hinzugefügt). Fällt schon in diesem Zitat auf, welchen Nachdruck Einstein auf die Verträglichkeit seiner Theorie mit allen bekannten Beobachtungen legte. so werden erst Recht die folgenden, in

\footnotetext{
${ }^{25} \mathrm{Vgl}$. vor allem Er. Stadler, Vom Positivismus zur 'wissenschaftlichen Weltauffassung'...(Wien und München, 1982), sowie H. Mulder, 'Wissenschaftliche Weltauffassung. Der Wiener Kreis', Journal of the History of Philosophy, 6 (1968), 386-390.

${ }^{26}$ Zu diesem Aspekt des logischen Empirismus vgl.z.B. die Einführungen von R. Hegselmann zu Schlicks 'Fragen der Ethik' (zit. in Anm. 3) und zu O. Neurath, Wissenschaftliche Weltauffassung, Sozialismus tnd logischer Empirismus (Frankfurt, 1979), 7-73.

${ }^{27}$ Originalarbeiten Einsteins, siehe Anm. 28 und 30, Eine populäre Einführung gab Einstein selbst in A Einstein, $\dot{U}$ ber die spezielle und die allgemeine Relativitätstheorie (Braunschweig, 1. Aufl. 1917, 21. Aufl. 1981).

${ }^{28}$ A. Einstein, 'Über den Einfluß der Schwerkraft auf die Ausbreitung des Lichtes', Annalen der Physik, (4) 35 (1911), 898-908 u. Reprints u.a. in: Lorentz, Einstein u. Minkowski (Anm. 8), 72-80.

${ }^{29}$ Nachdem eine nach Russland entsandte Expedition 1914 in die Wirren des 1. Weltkrieges geriet, gelang es erstmals 1919, den vorausgesagten Effekt nachzuweisen; vgl. dazu Dyson, Eddington u. Davidson, 'a determination of the deflection of light ..., Philosophical Transactions of the Royal Society of London, A, 110 (1920), 291-333.

${ }^{30}$ A. Einstein, 'Formale Grundlage der allgemeinen Relativitätstheorie', Sitzungsberichte der Preussischen Akademie der Wissenschaften, (1914), 2. Teil, S. 1030-85; ders, 'Grundgedanken der allgemeinen Relativitätstheorie und Anwendung dieser Theorie in der Astronomie, ebenda, (1915), 1. Teil, 315; 'Zur allgemeinen Relativitätstheorie', ebenda, (1915), 2. Teil, 778-86 u. 799-801; 'Erklärung der Perihelbewegung des Merkur aus der allg. Relativitätstheorie', ebenda, 831-9; 'Feldgleichungen der Gravitation', ebenda, S. 844 7; 'Die Grundlage der allgemeinen Relativitätstheorie', Annalen der Physik, 49 (1916), 769-822 u. Reprint in Lorentz, Einstein u. Minkowski (Anm. 8), S. 81-124.
} 
sarkastischer Selbstbescheidung verpackten Korrekturen der Theorielastigkeit der Schlickschen Darstellung erstaunen (ibid):

Mit der empirischen Kontrollierbarkeit der Theorie steht es nicht ganz so traurig, wie Sie angeben. Die Theorie erklärt die von Leverrier aufgefundene Perihelbewegung des Merkur quantitativ. Der von der Theorie geforderte Einfluß des Gravitationspotentials auf die Farbe des emittierenden Lichtes wurde durch die Astronomie bereits qualitativ bestätigt (Freundlich). Auch besteht gute Aussicht auf Prüfung des Resultates betr. die Krümmung der Lichtstrahlen durch das Schwerefeld. ${ }^{31}$

\section{Einfachheit}

Auch in seinem zweiten Aufsatz zur Relativitätstheorie hatte Schlick offensichtlich das Theorien-Verständnis Einsteins getroffen. Die Stellung der allg. Relativitätstheorie zur klassischen Mechanik umschrieb er 1917 z.B. so:

Eine allein auf relative Bewegungen aufgebaute Mechanik würde also ein sehr viel geschlosseneres, vollendeteres Weltbild ergeben, als die Newtonsche. Es wäre zwar nicht etwa (wie Mach meinte) als das einzig richtige Weltbild erwiesen, wohl aber empfähle es sich (wie Einstein hier hervorhebt) von vorneherein durch seine imposante Einfachheit und Abrundung. ${ }^{32}$

Auf diese Passage Bezug nehmend, schrieb Einstein in seinem Antwortschreiben nur zwei Tage (6 Februar 1917) nach Erhalt des Aufsatzes (!):

Ihre Darlegung ist von unübertrefflicher Klarheit und Übersichtlichkeit ... Wer Ihre Darlegung nicht versteht, der ist überhaupt unfähig, einen derartigen Gedanken aufzufassen. Sehr gut hat mir gefallen, daß Sie nicht a posteriori die allgemeine Relativitätstheorie als erkenntnistheoretisch notwendig, sondern nur als in höherem Masse befriedigend hingestellt haben. Zu kritisieren habe ich gar nichts, sondern nur die Treffsicherheit Ihres Denkens und Ihres Wortes zu bewundern'.

Hieraus ist ersichtlich, wie der Gedankenaustausch beider, schon sehr band nach seiner Aufnahme, sich konzentriert auf Fragen des Status physikalischer Hypothesen; diese selbst können, von gelegentlichen interpretatorischen Unsicherheiten Schlicks abgesehen, als geklärt angesehen werden. Der tiefere Grund für die geradezu emphatische Zustimmung Einsteins zu Schlicks Manuskripten und den danach erheblich intensivierten Kontakt zwischen beiden ist neben der Souveränität Schlicks in der Deutung

\footnotetext{
${ }^{31}$ Einstein war dann auch der erste, der den Erhalt eines Telegramms von Lorentz betreffend einer ersten Bestätigung seiner Lichtablenkungshypothese Publik machte, und zwar in folgender Notiz: A. Einstein, 'Prüfung der allgemeinen Relativitätstheorie', Die Naturwissenschaften, 7 (1919), 776. Die Zeilen aus dem Einstein-Brief an Schlick und dieser Umstand könnten dazu beitragen, der verbreiteten Legende vom Theoretiker Einstein, der such um experimentelle Prüfungen seiner Hypothesen nicht weiter sorgt, ein Ende zu setzen. Es ist dies ein gutes Beispiel für die vielfach nur in Privatbriefen zum Ausdruck kommenden Einschätzungen und Normen, deren Nicht-Berücksichtigung oftmals die Entstehung wissenschaftshistorischer Legenden mit sich bringt.

${ }^{32}$ M. Schlick, Raum und Zeit in der gegenwärtigen Physik.., Die Naturwissenschaften, 5 (1917 a), 161-7, 177-86; Berlin, 1. Aufl. 1917 b, 2. vermehrte Aufl. 1919, 4. verm. Aufl, 1922); Variantenverzeichnis in K. Hentschel (1984, Anm. 5), S. 199.

${ }^{33}$ Vgl. Schlick (1922, Anm. 20); Schlick (1929, ebenfalls zit. in Anm. 20) sowie M. Schlick, 'kritizistische oder empiristische Deutung der neuen Physik?", Kantstudien, 26 (1921), 96111.
} 
und Popularisierung des physikalischen Neulandes vor allem in dem ausdrücklichen Rekurs Schlicks auf das für Einstein so wichtige Motiv der 'Einfachheit' zu sehen. Schlick hatte es nicht nur verstanden, den naheliegenden aber irreführenden Fehlschlüssen der Common-Sense-Kommentatoren präzise Widerlegungen entgegenzustellen, ${ }^{33}$ sondern überdies Einsteins Konzept der Einfachheit ${ }^{34}$ aufgegriffen als das zwischen verschiedenen aber leistungsgleichen Theorien entscheidende Kriterium. ${ }^{35}$ Einstein wußte sehr wohl, daß eine Entscheidung zwischen seiner Relativitätstheorie und der damals noch von vielen protegierten Lorentz-Abrahamschen Variante der Elektrodynamik und verschiedenen Gravitationstheorien (Mie, Nordström, Jaumann...) ${ }^{36}$ nicht auf experimentellem Wege möglich sei; er wußte von der Möglichkeit ‘äquivalenter Beschreibungen' für ein und denselben Sachverhalt in verschiedenen Zeichensystemen-dennoch glaubte Einstein, die drohende Entscheidungslücke zwischen konkurrierenden Beschreibungen schließen zu können. Seiner Auffassung nach war die Relativitätstheorie vor allen anderen bis dato erdachten Konkurrenten dadurch ausgezeichnet, daß sie die bislang einfachst mögliche theoretische Erfassung aller bekannten Sachverhalte ermögliche. Dieses 'Zauberwort Einfachheit', dessen nähere Umschreibung in den Schriften Einsteins changiert zwischen 'Verständlichkeit, Schönheit, Natürlichkeit und Ökonomie (also Sparsamkeit der unabhängigen Grundannahmen bzw. -begriffe)' wurde von Schlick aufgegriffen und verwendet meist in der Bedeutungsschattierung 'Minimum an willkürlichen Elementen'. ${ }^{37}$ Während Einstein noch in späteren Schriften auf 'Einfachheitskriterien' zurückgriff, die ihm umproblematisch schienen, erkannte Schlick nach seiner anfänglichen Übernahme des Terminus (1917) später (ab 1925, verstärkt 1931ff) dessen grundlegende Vagheit und kam dann zu der Feststellung 'Einfachheit ist ein halb pragmatischer, halb ästhetischer Begriff' ${ }^{38}$ ohne mit dieser Feststellung die ihm wohlbekannte praktische Bedeutung irgendwie schmälern zu wollen. In dieser und in ähnlichen latenten Bedeutungsverschiebungen von zentralen Begriffen sind später zum Ausbruck gekommene Meinungsverschiedenheiten zwischen beiden bereits angelegt. Nur durch die Berücksichtigung der bislang der Öffentlichkeit unzugänglichen Korrespondenz und die hier nur angedeutete Analyse des Verwendungszusammenhangs der Termini ${ }^{39}$ war es möglich, die Wurzeln späterer Kontroversen in diese Phase zurückzuverfolgen.

\footnotetext{
${ }^{34}$ Vgl. dazu u.a. Elkana, 'The Myth of Simplicity', in Y. Elkana u. G. Holton (Anm. 2), S. 205-51.

${ }^{35}$ Ein typischer Beleg hierfür ist etwa folgende Stelle aus Schlick (1948, Anm. 22), S. 44: 'Es wäre möglich, einen absoluten Gleichzeitigkeitsbegriff aufrechtzuerhalten ... Demgegenüber ist die Einführung der relativen Gleichzeitigkeit viel einfacher und entspricht dem Prinzip, nur prinzipiell Beobachtbares in die Formulierung der Naturgesetze aufzunehmen'... (Hervorhebung hinzugefügt). Nicht zufällig ist auch in späteren Diskussionen über Theorienvergleich das Paradigma immer wieder Lorentz↔Einstein (vgl. Arbeiten von K. Schaffner und E. Zahar, 'Why did Einstein's programme supersede Lorentz'?', in Method and appraisal in the physical Sciences, hrsg. C. Howson (Cambridgc, 1976), S. 24-274 sowie die sich hieran anschließende Diskussion im British Journal for the Philosophy of Science.

${ }^{36}$ Vgl. z. B. A. I. Miller (1981, Anm. 7)

${ }^{37}$ Wobei die Ambiguität 'Elemente' = 'Grundbegriffe' oder 'Prinzipien' zusätzlich ungeklärt blieb; vgl hierzu Hentschel (Anm. 5) Kap. 4.

${ }^{38}$ M. Schlick, (Die Kausalität in der gegenwärtigen Physik', Die Naturwissenschaften, 19 (1931), 145-62, Zitat S. 148; vgl. eine analoge Passage in Schlick (1948, Anm. 22), S. 48

${ }^{39}$ Vgl. Hentschel (Anm. 5), Kap. 4, S. 49 ff.
} 


\section{4. 'Wirklich' in verschiedenem Sinne}

Noch im gleichen Jahr, 1917, treten diese latenten Unterschiede der Auffassung deutlicher zu Tage. Durch die ermunternden Worte Einsteins in dessen letztem Brief bestätigt, entschloss sich Schlick, die Arbeit über 'Raum und Zeit...' in erweiterter Fassung als ein kleines Buch herauszugeben. ${ }^{40}$ In ausdrücklicher Abgrenzung von Ernst Mach unterschied Schlick in einer Anmerkung des neu hinzukommenden Kapitels 'Beziehungen zur Philosophie'41 zwischen subjektivem Raum-Zeit-Erlebnis und dem objektivem Sinn von Raum und Zeit als Ordnungsschema physikalischer Vorgänge. Indem er dem 'strengen Positivismus eines Mach' unterstellt, daß darin 'der Begriff der physischen Welt in ihrer objektiven vierdimensionalen Ordnung tatsächlich nur ein abkürzender Ausdruck für die Korrespondenz der subjektiven raum-zeitlichen Erfahrungen verschiedener Sinnesgebiete und weiter nichts' sei, hält er diesem Zerrbild von Machs Monismus seine eigene Auffassung des 'kritischen Realismus' entgegen. Raum-zeitliche Ordnungsschemata sind für Schlick 1917 nicht nur ökonomische Hilfsbegriffe, die als abkürzende Sprechweise Gegebenes repräsentieren durch das Zeichensystem 'Naturwissenschaft' erkennen wir laut Schlick 'Realität'. Die Annahme, daß 'jeder für die Naturbeschreibung tatsächlich brauchbare Begriff auch in gleicher Weise als Zeichen für etwas Wirkliches betrachtet werden darf' erscheint Schlick für eine 'wohlgerundete', gleichsam 'löcherfreie' Weltansicht unentbehrlich. ${ }^{42}$ Insgesamt interessiert Schlick sich von nun an mehr und mehr für den Bezeichnungsvorgang und das netzartige Zusammenwirken von Zeichen in Beschreibungen und Theorien. ${ }^{43}$

Im Mai 1917 reagiert Einstein auf diese Ausführungen überraschend kritisch in einer Form, wie sie paradoxerweise schon deutlich das spätere Selbstverständnis Schlicks (der Philosoph als neutraler Begriffsklärer und Sinndeuter) ${ }^{44}$ vorwegnimmt, welches Schlick in den dreißiger Jahren unter dem Einfluß Wittgensteins entwickelte, Es folgt ein längeres, wegen seiner Bedeutung und Prägnanz von mir ungekürztes Zitat aus dem Brief Einsteins am 21 Mai 1917.

Es scheint mir nun, daß das Wort 'wirklich' in verschiedenem Sinne genommen wird, je nachdem es von Empfindungen oder von Ereignissen bzw. Thatbeständen in physikalischem Sinne ausgesprochen wird.

Wenn zwei verschiedene Völker unabhängig voneinander Physik treiben, werden sie Systeme schaffen, die bezüglich der Empfindungen ('Elemente' im Sinne Machs) ${ }^{45}$ gewiss übereinstimmen. Die gedankliche Konstruktion, die die beiden zur Verknüpfung dieser 'Elemente' ersonnen, können weitgehend verschieden sein. Beide Konstruktionen brauchen auch nicht übereinzustimmen bezüglich der 'Ereignisse'; denn diese gehören sicherlich zu den begrifflichen

${ }^{40}$ M. Schlick (1917 b, Anm. 32).

${ }^{41}$ Ebenda, S. $51 f f$ (1. Aufl.), S. $73 f f$ (2. Aufl.).

${ }^{42}$ Ebenda, S. $80 \mathrm{ff}$ (2. Aufi.).

${ }^{43} \mathrm{Vgl}$. dazu insb. die Ergänzungen von Schlicks Allgemeiner Erkenntnislehre (zit Anm. 3) in deren 2. Auflage 1925 .

${ }^{44} \mathrm{Vgl}$. Anm. 21 und 23 sowie die Briefe Schlicks an Einstein vom 5 Juni 1927 und 14 Juli 1927, in denen Schlick begeistert über seine Studien in 'neuer Logik' (gemeint sind Frege, Russell, Whitehead und Wittgenstein) berichtet und u.a. schreibt, er erwarte sich von Wittgensteins Philosophieren eine 'gänzliche Reform der Philosophie'.

${ }^{45}$ Eine Anspielung auf Machs Terminologie in E. Mach, Analyse der Empfindungen (Jena, 1. Aufl. 1886, 5. Aufl. 1906), S. 4, 7 etc. 
Konstruktionen. Wirklich im Sinne von 'in der Erfahrung unabweislich gegeben' sind gewiss nur die 'Elemente', nicht aber die 'Ereignisse'.

Bezeichnen wir aber als 'wirklich' das in Raum und Zeitschema von uns Eingeordnete, wie Sie es in der Erkenntnistheorie gethan haben, so sind in erster Linie zweifellos die 'Ereignisse' wirklich.

Nach dieser Exposition der beiden konkurrierenden Konstruktion von Wirklichkeit kommt Einstein zu einer bemerkenswerten Fesstellung dessen, was ich 'erkenntnistheoretischen Konventionalismus ${ }^{\mathbf{1 4 6}}$ genannt habe: beiden Sprechweisen werden Vor- und Nachteile zugesprochen - in begrifflicher Umsicht, nicht in der Entscheidung für eine der beiden sieht Einstein die Afugabe. Die Entscheidung, die jeder einzelne letztendlich trifft, tritt in ihrer Bedeutung zurück gegenüber der jeweiligen Analyse des Verwendungszusammenhangs und der Ziele, die man vor Augen hat: 'Was wir nun in der Physik als "wirklich" bezeichnen, ist zweifellos das "zeiträumlich Eingeordnete", nicht das "Unmittelbar-Gegebene". Das Unmittelbar-Gegebene kann Illusion sein, das Zeiträumlich-Eingeordnete kann ein steriler Begriff sein, der nichts zur Aufhellung der Zusammenhänge zwischen dem Unmittelbar-Gegebenen beiträgt. Ich möchte hier eine reinliche Begriffsscheidung vorschlagen". (ibid.) Es darf am Ende dieses Unterabschnittes der Ausblick auf die weitere Entwicklung der von Einstein und Schlick vertretenen Positionen nicht fehlen, da diese sich auf merkwürdige Weise überkreuzen sollten. Nach etlichen fehlgeschlagenen Versuchen an anderen Universitäten ${ }^{47}$ gelang es Schlick im Jahr 1922 (nach einer kurzen Übergangszeit in Kiel) ${ }^{48}$ einen Lehrstuhl an der Universität Wien zu bekommen, ${ }^{49}$ wo er sehr bald zur Zentralfigur des sogenannten 'Wiener Kreises' wurde. ${ }^{50}$

Im Zuge der dortigen Diskussionen vertrat Schlick dann zeitweise (besonders deutlich in der sogenannten Idealismus-Realismus-Debatte) ${ }^{51}$ die Position, da 3 alle 'ontologischen Aussagen', ob solche des Realismus, Idealismus oder Positivismus gleichermaßen als 'metaphysisch' zu betrachten und deshalb $\mathrm{zu}$ umgehen seien, ${ }^{52}$ womit er sich dem scheinbar voraussetzungsfreien Sprechweisen-Vergleich, wie er im Einstein-Brief Ende Mai 1917 vertreten worden war, stark annäherte. Umgekehrt bekannte sich Einstein nach 1920 und insb. in seinen Diskussionen mit Vertretern der

${ }^{46} \mathrm{Vgl}$. Hentschel (Anm. 5) Kap. 6.

${ }^{47}$ Wie aus den Briefen vom 22 Februar 1920, 28 Februar 1920, 22 April 1920, 10 Mai 1920, 29 August 1920, 13 August 1922 hervorgeht, waren erwogen worden Lehrstühle in Zürich, Gießen, Prag, dann Kiel, wo Schlick ab 1921 dozierte, und schließlich Wien (ab 1922)

${ }^{48}$ Im Jahr 1921 vgl. Anm. 47.

${ }^{49}$ Ab 1922. Schlick wurde berufen auf den Lehrstuhl, den vor ihm (bis '01) Ernst Mach und dann Ludwig Boltzmann (-1906) und Adolf Stöhr (-1921) innehatten.

${ }^{50} \mathrm{Vgl}$. dazu Zitate in Anm. 25 sowie etwa V. Kraft, Der Wiener Kreis, der Ursprung des Neopositivismus. Ein Kapitel jüngster Philosophiegeschichte (Wien, 1950).

${ }^{51}$ Vgl. u.a. M. Schlick, 'Positivismus und Realismus', Erkenntnis, 3 (1933), 1-31; R. Carnap, Scheinprobleme in der Philosophie. Das Fremdpsychische und der Realismusstreit (Berlin, 1928); H. Feigl, Theorie und Erfahrung in der Physik (Karlsruhe, 1929); O. Neurath, 'Radikaler Physikalismus und 'wirkliche Welt', Erkennmis. 4 (1934), 346 62: Schlick (1948. Anm. 22). S. 34 t

${ }^{52}$ Vgl. Schlick (1933, Anm, 51), 4. 28. 30. 
Kopenhagener Interpretation der Quantenmechanik Ende der zwanziger und in den dreißiger Jahren ${ }^{53}$ immer dezidierter zur Position des 'Realismus', die Schlick ursprünglich vertreten hatte. ${ }^{54}$

Auch in der Korrespondenz mit Schlick finden Einsteins QuantenmechanikDebatten Eingang. Nachdem Schlick in verschiedenen Aufsätzen den radikal veränderten Wirklichkeitsbegriff der Quantenmechanik vorgestellt hatte, ${ }^{55}$ reagierte Einstein am 26 November 1930 wie folgt:

Allgemein betrachtet entspricht Ihre Darstellung insofern nicht meiner Auffassung, als ich Ihre ganze Auffassung sozusagen zu positivistisch finde ${ }^{56} \ldots$. Ich sage Ihnen glatt heraus: die Physik ist ein Versuch der begrifflichen Konstruktion eines Modells der realen Welt sowie von deren gesetzlicher Struktur. Allerdings muß sie die empirischen Relationen zwischen den uns zugänglichen Sinnenerlebnissen exakt darstellen; aber nur so ist sie an letztere gekettet ${ }^{57}$... Sie werden sich über den 'Metaphysiker' Einstein wundern, aber jedes vier- und zweibeinige Tier ist in diesem Sinne de facto Metaphysiker.

Mit dieser schroffen Absage Einsteins an Schlicks Ansatz, die hier zeitlich vorausgreifend angeführt wurde, war der konstruktive Teil des Gedankenaustausches zwischen beiden natürlich abgeschlossen-zu fundamental waren die Differenzen geworden, als daß sich mehr denn wechselseitiger Bericht über die eigenen Aktivitäten und die Klärung sach- bzw. personenbezogener Angelegenheiten (wie insb. der Berufungsfrage Hans Reichenbach) ${ }^{58}$ hätte anschließen können.

\section{Newton und die Kausalität}

Nur noch ein weiterer Themenbereich aus der großen Fülle behandelter Probleme soll hier ausführlicher behandelt werden: in einer Debatte, die die Einstein-SchlickKorrespondenz vom März 1917-August 1920 prägt, also noch deutlich vor der im vorigen bereits erwähnten Entwicklung, diskutierten beide die gleichermaßen wissenschaftshistorisch wie -systematisch interessante Frage, $o b$ bzw. inwieweit die klassische Mechanik Newtonscher Prägung ${ }^{59}$ die Forderungen des Kausalitätsprinzips erfülle und in welchem Sinne die Weiterentwicklung der Physik bis zur Relativitätstheorie in

\footnotetext{
${ }^{53}$ Vgl. u.a. A. Einstein, 'Electron et photon', Rapports et discussions du Sème conseil Solvay (1927, Paris), S. 253-6; 'Can quantum mechanical description of physical reality be considered complete?, Physical Review, 47 (1935), 777-80; 'Quantenmechanik und Wirklichkeit', Dialectica, 2 (1948), 320-4.

${ }^{54}$ Einstein (1935), zitiert in Anm. 53, verfasst von B. Podolsky, zusammen mit Einsten und N. Rosen beginnt mit einer Definition dessen, was unter 'Realität' zu verstehen sei; typisch für diese Form der Darstellung sind auch die 'Einleitende(n) Bemerkungen über Grundbegriffe' Einsteins in Louis de Broglie Physicien 't l'nseur (Paris, 1953), S. 4-15.

" $\backslash$ gl. Sihlick, 'Die Kausalität in der gegenwärtigen Physik', Die Naturwissenschaften, 19(1931), 145-62 sowie später M. Schlick, 'Quantentheorie und Erkennbarkeit der Natur', Erkenntnis, 6 (1936-37), 317-26.

${ }^{56}$ Ein Vorwurf, der deshalb von besonderer Ironie war, weil Schlick sich seit seiner Allgemeinen Erkenntnislehre selbst als Kritiker des Positivismus verstand; vgl. \$\$ 35, 32, 26 a von Schlick (1918, Anm. 3).

${ }^{57}$ Einstein schilderte 'seine' Erkenntnistheorie besonders eingehend in einem Brief an Solovine; vgl. Lettres à Maurice Solovine (Paris, 1956), S. 120; kommentiert auch in Holton (1981, Anm. 2), S. 372-418.

${ }^{58}$ Wie aus den Briefen vom 27 Dezember 1925, 1 Februar 1926 an Einstein und einigen Briefen der Korrespondenz Schlick-Planck ersichtlich ist, traten Planck und Einstein als Fürsprecher auf in der Diskussion darum, ob Hans Reichenbach die Möglichkeit der Habilitation gegeben werden sollte, obwohl bekannt geworden war, daß dieser in seiner studentischen Jugend despektierliche Pamphlete gegen Militarismus und Gelehrtendünkel verfasst hatte; vgl. hierzu: H. Reichenbach, 'Student und Sozialismus', Der Aufhau. Flugblätter an Jugend, o.J. (1919) Bd. 5; vgl. a. H. Hecht u. D. Hoffmann, 'Die Berufung H. Reichenbachs an die Berliner Universität', Deutsche Zeitschrift für Philosophie, 30 (1982), 651-62.

${ }^{59} \mathrm{Vgl}$. I. Newton, Mathematische Prinzipien der Naturlehre (1687), dt. Ubersetzung J. Ph. Wolfers (Berlin 1872. Reprint Darmstad1. 1963).
} 
diesem Sinne als ein 'Fortschritt' aufzufassen sei. Der Ausgangspunkt der Kontroverse war diesmal eine Anmerkung, die Einstein zum Manuskript des Aufsatzes über 'Raum und Zeit...' von Schlick gemacht hatte und die unverändert in deren Druck übernommen worden war: 'Einstein fügt hinzu, die Newtonsche Mechanik leiste z.B. in Bezug auf den Fall des rotierenden, sich abplattenden Körpers der Forderung der Kausalität nur scheinbar Genüge'.60

Der nicht ausgesprochene Argumentationsgang, der zur Stützung dieser Einsteinschen Behauptung herangezogen werden sollte, um sie verständlich zu machen, sähe etwa wie folgt aus. Die 'Erklärung' Newtons für die Abplattung eines rotierenden Körpers, der im Ruhezustand als Kugel vermessen worden ist, führt als 'Ursache' für diese Abplattung an, daß immer, wenn ein Körper in Bezug auf den absoluten Raum beschleunigt werde, Kräfte aufträten, die die Verzerrung der Gestalt des Körpers herbeiführten. ${ }^{61}$ Die Scheinhaftigkeit dieser Kausalerklärung liegt für Einstein in dem Rekurs Newtons auf einen nicht nachweisbaren absoluten Raum ${ }^{62}$ das eine, zu erklärende Phänomen werde gleichsam auf ein noch weniger konkretes verschobendie behauptete Erklärung sei nur geeignet, das Phänomen, dem sie ihre Erfindung verdanke, abzudecken, ohne dass eine weitere Einbindung der Hypothese in das theoretische Netzwerk erfolge. Demgegenüber würde seine (Einsteins) Hypothese der Wirkung des Gravitationsfeldes auf die Raum-Zeit-Struktur eine 'echte Erklärung' dieses Sachverhaltes darstellen--die auftretende Hypothese (Krümmung der RaumZeit-Struktur) fungiere bei seiner Theorie als Ursache $u$ nd als Wirkung, nicht einseitig nur als Ursache. ${ }^{63}$

Schlick war von dieser Argumentation anfangs nicht überzeugt. An die zitierte Einsteinsche Anmerkung in seinem Text fügte er folgende Ergänzung an:

Diese Formulierung erscheint mir aber nicht ganz einwandfrei. Man braucht die Newtonsche Lehre wohl nicht so aufzufassen, als erkläre sie den Galileischen Raum, der ja freilich keine beobachtbare Sache ist, für die Ursache der Zentrifugalkräfte, sondern man kann die Redeweise vom absoluten Raum wohl auch als die Umschreibung der bloßen Tatsache des Daseins dieser Kräfte betrachten. ${ }^{64}$

Auf diese, in der Aufsatzanmerkung eingefügte Erwiderung reagierte Einstein zunächst in seinem Brief an Schlick 21 März 1917 mit einem scheinbaren Zugeständnis:

Mit Ihrer Kritik ... haben Sie Recht. Die Forderung der Kausalität ist eben bei genauem Hinsehen keine scharf umgrenzte. Es gibt verschiedene Grade der Erfüllung der Kausalitätsforderung. Man kann nur sagen, daß die Erfüllung der allgemeinen Relativitätstheorie in höherem Maße geglückt ist als der klassischen Mechanik. Die sorgfältige Durchführung dieses Gedankens wäre vielleicht eine lohnende Aufgabe für einen Erkenntnistheoretiker.

\footnotetext{
${ }^{60}$ Schlick verweist an dieser Stelle dann auf Einsteins Abhandlung aus dem Jahr 1916, zitiert in Anm. 30: Annlen der Physik, 49, 771.

${ }^{61}$ Newton (1687/1872), zitiert in Anm. 59), S. 28 f. (Anm. zur Erklärung 8).

${ }^{62}$ Ibid, II. der Anm. zur Erklärung 8.

${ }^{63}$ Eine populäre Erklärung und Erläuterung des Sachverhaltes, den Einstein hier engedeutet hat, gab er in Einstein (1917, zitiert in Anm. 27), S. 114 ff., 125 f.: 'Gemäß der allgemeinen Relativitätstheorie dagegen hat der Raum gegenüber dem 'Raum-Erfüllenden' keine Sonderexistenz' (ibid.).

${ }^{64}$ In Schlick (1917 a, Anm. 32), 178 sowie Schlick (1948, Anm. 22), S. 35.
} 
Mit dieser Umschreibung der Erwiderung Schlicks zeigt sich jedoch, daß Einstein an dieser Stelle den 'linguistic turn' der Schlickschen Argumentation verkannte. Es ging Schlick nicht um eine Differenzierung verschiedener Grade der Erfüllung der Kausalitätsforderung, sondern um eine grundlegend andere Interpretation des Kausalitätsprinzips. Kausalität war für Schlick nicht mehr eine Beziehung zwischen Gegebenem, sondern lediglich eine Relation zwischen Zeichen für Gegebenes. Das, was durch die realistische Interpretation der benutzten Termini zur Unterstellung einer kausalen Beziehung zweier Phänomene zueinander wird, ist für Schlick lediglich eine sprachliche Beziehung zweier Elemente des Zeichenvorrates untereinander. In Schlicks Worten aus dem Jahr 1932: 'Das Kausalprinzip ist nicht selbst ein Gesetz, sondern will nur das Bestehen von Gesetzen ausdrücken'; ${ }^{65}$ es ist also kein Satz über Tatsachen, sondern nur über die Form von Gesetzen über Naturprozesse. Die von derart grundsätzlichen Mißverständnissen durchzogene Debatte hierüber zieht sich durch die Korrespondenz bis zum Brief Einsteins vom Monatsende Juni 1920, in dem er seine Auffassung in dieser 'schwierigen Angelegenheit' folgendermaßen vertrat: In Newtons Grundgleichung (Kraft gleich Masse mal Beschleunigung) seien Kraft und Masse 'absolut' definiert. Aus Konsistenzgründen sei es deshalb erforderlich, daß auch die Beschleunigung, definiert als zweite Ableitung des Ortes nach der Zeit einen 'absoluten Sinn' erhalte. Er würdigt diese Konsequenz, zu der Newton sich durchgerungen habe, folgendermaßen: 'Es ist für Newtons logisches Gewissen höchst ehrenvoll, dass er sich zur Schöpfung des absoluten Raumes (und der absoluten Zeit, was allerdings weniger wichtig war) entschloss. Er hätte den absoluten Raum ebensogut "starrer Äther" nennen können. Er brauchte eine solche Realität, um der Beschleunigung einen objektiven Sinn zu geben' und in einer Art Überhöhung dieser 'Ehrenrettung' des von vielen Einstein-Epigonen gerupften Newton führte Einstein weiter aus: 'die späteren Versuche, ohne diesen absoluten Raum in der Mechanik auszukommen, waren (bis auf den Machschen) ${ }^{66}$ nur "Versteckspielen"' (31 Juni 1920).

Es ist bemerkenswert, daß ausgerechnet Einstein, dem von Seiten des logischen Empirismus immer wieder bescheinigt wurde, mustergültig 'abgestandene metaphysische Konzepte' (wie hier den absoluten Raum) aus der Naturwissenschaft 'verbannt zu haben", ${ }^{67}$ daß ausgerechnet Einstein also die Einführung dieses Konzeptes durch Newton als den 'größten Schritt, den das Streben nach kausaler Verkettung der Naturerscheinungen je gemacht hat' würdigt und retrospektiv rechtfertigt als die in seiner historischen Situation einzig konsequente Haltung. ${ }^{68}$

Die Diskussion um die Frage, ob Newton 'echte' Kausalerklärungen abgegeben hat, ist aufschlußreich auch für das von Einstein bzw. Schlick implizit unterstellte Modell (wissenschafts) geschichtlicher Entwicklungen, insb., wenn man es noch um das im vorigen zum Einfachheitsprinzip und seiner Funktion in der Argumentation

\footnotetext{
${ }^{65} \mathrm{Vgl}$. Schlick (1931, zit in Anm. 55) sowie Schlick (1948, zit in Anm. 22) Zitat aus Schlick (1948), S. 47; man vergleiche hiermit die Sätze 6.32, 6.33, 6.34, 6.35, 6.36 und deren Untersätze in Wittgensteins Tractatus, zitiert in Anm. 23.

${ }^{66}$ Eine Anspielung Einsteins auf Ernst Mach, Die Mechanik in ihrer Entwicklung historisch-kritisch dargestellt (Leipzig, 1. Aufl. 1883), 2. Kapitel, Abschnitt 6 und 7.

${ }^{67}$ Eine typische Abhandlung diesen Charakters ist etwa H. Reichenbach, Von Kopernikus bis Einstein (Berlin, 1927); vgl, auch Schlicks in Anm. 71 zitierten Aufsatz.

${ }^{68}$ Eine analoge Passage findet sich z.B. in Einstein (1917), zit in Anm. 27, S. 107f. sowie in A. Einstein, 'Newtons Mechanik und ihr Einfluß auf die Gestaltung der theoretischen Physik', Die Naturwissenschaften, 15 (1927), 273-6.
} 
Einsteins bzw. Schlicks Gesagte ergänzt. In Bezug auf die Mechanik Newtons und deren historisches Verhältnis zur allgemeinen Relativitätstheorie setzt Einstein in seinen Darstellungen den Akzent auf die graduelle Wandlung der Erfüllung bestimmter übergeordneter Forderungen (wie Denkökonomie, Einfachheit oder Kausalitätsprinzip). D.h. Fortschritt der Wissenschaften bedeutet für Einstein, mindestens für die Zeit nach Galilei, die Aneinanderreihung unzähliger, kleiner, fast selbstverständlicher Gedankenschritte unter der Randbedingung der Erreichung immer einheitlicherer, einfacherer, weitreichenderer Behandlung der Naturphänomene. 'Die ganze Entwicklung unserer Ideen über das Naturgeschehen ... könnte als eine organische Fortbildung Newtonscher Gedanken aufgefasst werden' ${ }^{69}$ Auch seine eigenen Leistungen bezog Einstein willig in diese Kontinuitätsthese mit ein: 'The ascent of relativity is composed of small, almost self-evident steps of thought'. ${ }^{70}$

Umgekehrt hat Moritz Schlick, gerade in seinen populären Aufsätzen über Einsteins Relativitätstheorie die Neuartigkeit, den revolutionären Charakter der Einsteinschen Neuerungen in der Mechanik und Elektrodynamik hervorgehoben, so daß bei ihm vor Einstein nur Copernikus 'einen Hauch des Geistes durch die Gefilde des Naturerkennens wehen ließ und die vertrockneten Reste überwundener Weltansichten reinigend' hinwegblies. ${ }^{71}$

Wegen der eigenartigen Mischung historischer Diskussionsgegenstände mit systematischen Problemen (Newtons Mechanik resp. 'echte Erklärung') betrieben beide letztendlich nicht historisch-hermeneutische, sondern systematische (wissenschaftstheoretische) Rekonstruktion.

\section{Anerkennungen}

Für die Publikation des vollständigen Briefwechsels beider sei der Leser verwiesen auf die chronologisch organisierten Korrespondenzbände der bei Princeton University Press erscheinenden Collected papers of Albert Einstein (Herausgeber J. Stachel) bzw. auf eine von den Verwaltern der Vienna Circle Foundation, Amsterdam geplante Edition der erhaltenen Schlick-Korrespondenz bei Suhrkamp. Letzteren sei gedankt für die Möglichkeit der Einsichtnahme in die Schlick-Papiere sowie für die Erlaubnis, die bisher unveröffentlichten Dokumente zu publizieren. Ebenfalls danke ich der Library of the Hebrew University, Jerusalem für die Veröffentlichungsgenehmigung betr. Einstein-Dokumente. Ferner danke ich Herrn Professor A. Kleinert (Institut für Geschichte der Naturwissenschaften, Hamburg) und Herrn Professor L. Schäfer (Philosophisches Seminar, Hamburg) für die Betreuung meiner bisherigen Studien.

\footnotetext{
${ }^{69}$ Ebenda, 276.

${ }^{70} \mathrm{~A}$. Einstein, 'Fundamental ideas and problems of the theory of relativity', Vorlesung, gehalten am 11 Juli 1923, Nobel lectures...(Amsterdam, 1967), S. 482-90 im Band 'Physics, 1901-1921'.

${ }^{71}$ M. Schlick, 'Einsteins Relativitätstheorie', Mosse-Almanach (Berlin, 1921), S. 105-23 (S. 106).
} 